\title{
STRONG CONVERGENCE IN A PRODUCT SPACE
}

JAMES SERRIN

The purpose of this note is to present a certain necessary and sufficient condition for strong convergence in $L_{p}$ over a product space. This result is formulated as Theorem 1 below. Theorem 1 includes as a special case a well-known compactness condition of M. Riesz, which we state in slightly different form as Theorem 2 . In the final part of the paper we show that Theorem 1 can be used to give very simple proofs of the so-called Rellich lemma of functional analysis.

1. Weak and strong convergence on product spaces. Let $S=E^{n} \times E^{m}$ be the cartesian product of the Euclidean number spaces $E^{n}$ and $E^{m}$. Points in $S$ will be denoted by $z$. Also we shall frequently write $z=(x, y)$, where $x \in E^{n}$ and $y \in E^{m}$ are the orthogonal projections of $z$ onto $E^{n}$ and $E^{m}$, respectively.

Consider functions $u=u(z)$ of class $L_{p}$ on $S$, where $1 \leqq p<\infty$. The following norms will be used,

$$
\|u\|=\left(\int|u(z)|^{p} d z\right)^{1 / p}, \quad\|u\|_{x}=\left(\int|u(x, y)|^{p} d x\right)^{1 / p} .
$$

The integrations here are over $S$ and $E^{n}$ respectively, and $\|u\|_{x}$ is of course itself a function of class $L_{p}$ over $E^{m}$.

THEOREM 1. Let $\left\{u_{k}\right\}$ be a sequence of functions of class $L_{p}(S)$, having joint compact support in S. Suppose that for almost all fixed $y$ the functions $u_{k}(x, y)$ converge weakly in $L_{p}\left(E^{n}\right)$, and that

$$
\left\|u_{k}\right\|_{x} \leqq v(y), \quad k=1,2, \cdots,
$$

where $v(y)$ is of class $L_{p}\left(E^{m}\right)$. Then the sequence $\left\{u_{k}\right\}$ converges strongly in $L_{p}(S)$ if and only if

$$
\lim _{|t| \rightarrow 0}\left\|u_{k}(x+t, y)-u_{k}(x, y)\right\|=0
$$

uniformly in $k$.

Proof. Let $\tilde{K}(\xi)$ be a non-negative continuous function on $E^{n}$, with support in $|\xi| \leqq 1$, and such that $\int \tilde{K} d \xi=1$. For each positive real number $h$ we set

$$
K(\xi)=K(\xi, h)=h^{-n} \tilde{K}(\xi / h)
$$

Received by the editors April 28, 1961. 
and define an integral average of functions $u \in L_{p}(S)$ by the formula

$$
u_{h}=u_{h}(z)=\int K(\xi-x) u(\xi, y) d \xi .
$$

By Hölder's inequality we have

$$
\begin{aligned}
\left|u_{h}-u\right|^{p} & \leqq \int K(\xi-x)|u(\xi, y)-u(x, y)|^{p} d \xi \\
& \leqq \text { Const. } h^{-n} \int_{|t| \leqq h}|u(x+t, y)-u(x, y)|^{p} d t .
\end{aligned}
$$

Now suppose $\left\{u_{k}\right\}$ is a sequence of functions satisfying the hypotheses of Theorem 1, and such that condition (2) holds. Then taking account of (2) and (3) one obtains

$$
\lim _{h \rightarrow 0}\left\|u_{k h}-u_{k}\right\|=0, \quad \quad \text { uniformly in } k .
$$

Let $u_{0}(x, y)$ denote the weak limit of the functions $u_{k}(x, y)$. Suppose for the moment that $u_{0}$ is in $L_{p}(S)$, which will certainly be the case if, for example, $u_{0}$ is measurable on $S$. Then we have

$$
\left\|u_{k}-u_{0}\right\| \leqq\left\|u_{k}-u_{k h}\right\|+\left\|u_{k h}-u_{0 h}\right\|+\left\|u_{0 h}-u_{0}\right\| .
$$

Let $\epsilon>0$ be fixed, and choose $h$ so small that the first and last terms on the right-hand side of (5) are each less than $\epsilon / 2$; in view of (4) this choice can clearly be made independent of $k$. Therefore

$$
\lim \left\|u_{k}-u_{0}\right\| \leqq \epsilon+\lim \left\|u_{k h}-u_{0 h}\right\| .
$$

It follows that $\left\{u_{k}\right\}$ will converge strongly to $u_{0}$ provided the righthand limit is zero. But

By the hypothesis of weak convergence, $u_{k h} \rightarrow u_{0 h}$ for almost all $z$.

$$
\left|u_{k h}\right|^{p} \leqq \text { Const. } h^{-n} \int\left|u_{k}(x, y)\right|^{p} d x \leqq \text { Const. } h^{-n_{v^{p}}},
$$

so that $\left|u_{k h}-u_{0 h}\right|^{p}$ is dominated by a fixed summable function (here we use the fact that the functions $u_{k}$ have joint compact support). The required conclusion thus follows from Lebesgue's theorem.

If $u_{0}$ is not originally known to be in $L_{p}(S)$, then a slightly modified version of the above argument may be used to show that $\left\{u_{k}\right\}$ is Cauchy in $L_{p}(S)$; we shall omit the details since they add nothing essentially new. The first half of the theorem is therefore proved. To complete the demonstration it is only necessary to show that (2) 
holds if $\left\{u_{k}\right\}$ is strongly convergent. This is, however, a consequence of the well-known fact that (2) holds for fixed $k$, together with the argument given on p. 100 of [3].

By taking $m=0$ in the preceding theorem we immediately obtain the following corollary result.

THEOREM 2. Let $\left\{u_{k}\right\}$ be a sequence of functions of class $L_{p}\left(E^{n}\right)$, having joint compact support. Suppose that $\left\{u_{k}\right\}$ is weakly convergent in $L_{p}\left(E^{n}\right)$. Then $\left\{u_{k}\right\}$ is strongly convergent if and only if

$$
\lim _{|t| \rightarrow 0}\left\|u_{k}(x+t)-u_{k}(x)\right\|=0 \quad \text { uniformly in } k .
$$

Theorem 2 implies a well-known compactness condition of $\mathrm{M}$. Riesz (see [5], or [2, Theorem IV. 8.21]). In fact, for the case of joint compact support and $1<p<\infty$ the latter theorem follows at once from Theorem 2 and the fact that $L_{p}$ is weakly compact. For $p=1$ a slight additional argument is required which we shall omit. The hypothesis of joint compact support in both Theorems 1 and 2 can easily be replaced by a uniformity condition for large $x$, as in [2] or [5].

2. Rellich's lemma. An important application of Theorems 1 and 2 is in showing that certain spaces of differentiable functions are compact. We present two such results here. The first is a slight generalization of Rellich's lemma (cf. [1, p. 489]), and the second is an extension of a similar but lesser known result of $E$. Hopf.

TheOREM 3. Let $K$ be a family of differentiable functions $u=u(x)$ with joint compact support. Suppose the norm $\|D u\|_{p}$ is uniformly bounded in $K$, where $p \leqq n .^{1}$ Then $K$ is compact in $L_{q}$ for $1 \leqq q$ $<n p /(n-p)$.

Proof. It is enough to consider any fixed $q$ satisfying $1<q$ $<n p /(n-p)$. By standard and well-known arguments we obtain the inequality

$$
\|u(x+t)-u(x)\|_{q} \leqq \text { Const. }|t| \alpha\|D u\|_{p}, \quad u \in C^{1},
$$

where $\alpha=1+n / q-n / p>0$, and the Const. depends only on $p, q$, and $\boldsymbol{n}$. (In the important case $q=p$ inequality (7) specializes to

$$
\|u(x+t)-u(x)\|_{p} \leqq|t| \cdot\|D u\|_{p}
$$

as can be seen at once by integration of the inequality

$1\|f\|_{p}$ denotes the usual $L_{p}$ norm of $f(x)$ on $E^{n}$. When $p>n$ the functions $u$ are well known to be uniformly Hölder continuous (C. B. Morrey, Jr., University of California Publ. Math. 1943, p. 13), and Ascoli's compactness theorem applies. 


$$
\left.|u(x+t)-u(x)| \leqq|t| \cdot \int_{0}^{1}|D u(x+\sigma t)| d \sigma .\right)
$$

It now follows from (7) that $\|u\|_{q}$ is uniformly bounded in $K$, whence $K$ is weakly compact in $L_{q}$. But then by virtue of $(7)$ and Theorem 2, $K$ is strongly compact in $L_{q}$. Q.E.D.

Whether $K$ is compact in $L_{q}$ for $q=n p /(n-p)$ is an open question, but the author is inclined to doubt that this is true. The special case $m=1, p=2$, of the following theorem is due to E. Hopf.

THEOREM 4. Let $\left\{u_{k}\right\}$ be a sequence of differentiable functions $u_{k}(z)$, having joint compact support in $S$. Suppose that for almost all fixed $y$ the functions $u_{k}(x, y)$ converge weakly in $L_{p}\left(E^{n}\right)$ and that (1) holds. Finally, assume that the norms $\left\|D u_{k}\right\|$ are uniformly bounded, the differentiations being taken with respect to the space $E^{n}$. Then $\left\{u_{k}\right\}$ is strongly convergent in $L_{p}(S)$.

Proof. It is enough to show that condition (2) of Theorem 1 holds for the sequence $\left\{u_{k}\right\}$. But by $\left(7^{\prime}\right)$,

$$
\left\|u_{k}(x+t, y)-u_{k}(x, y)\right\|_{x} \leqq|t| \cdot\left\|D u_{k}\right\|_{x}
$$

whence in tegrating over $E_{m}$ we get $\left\|u_{k}(x+t, y)-u_{k}(x, y)\right\| \leqq$ Const. $|t|$, and the proof is complete.

Added in proof. Theorems similar to those above are easily proved for functions in the space $L_{p, p^{\prime}}(S)$, that is, functions for which the norm

$$
\left(\int_{E^{m}}\left(\int_{E^{n}}|f(x, y)|^{p} d x\right)^{p^{\prime} / p} d y\right)^{1 / p^{\prime}}<\infty .
$$

In fact, Benedek and Panzone have obtained analogous results even for the class $L^{P, P^{\prime}}$ (the notation is apparent from their paper, The spaces $L^{P}$, with mixed norms, Duke Math. J. (1961)), and for functions with values in a Banach space.

Benedek and Panzone have also kindly communicated an example showing that the conjecture following the proof of Theorem 3 is correct. Indeed, consider the sequence of functions

$$
f_{k}(x)= \begin{cases}k^{(n-p) / p}(1-k|x|) & |x|<1 / k, \\ 0 & |x| \geqq 1 / k,\end{cases}
$$

where $1 \leqq p<n$. Then evidently $\left\|D f_{k}\right\|_{p} \equiv$ Constant, $k=1,2, \cdots$. On the other hand, if we put $q=n p /(n-p)$, then $\left\|f_{k}\right\|_{q} \equiv$ Constant. Since $f_{k} \rightarrow 0$ almost everywhere, it follows that the sequence $\left\{f_{k}\right\}$ cannot be compact in $L_{q}$. 
Note. The above research was partially supported by the United States Air Force under Contract AF 49(638)-262.

\section{REFERENCES}

1. R. Courant and D. Hilbert, Methoden der mathematischen Physik, Vol. II, Springer, Berlin, 1937.

2. N. Dunford and J. Schwartz, Linear operators, Interscience, New York, 1959.

3. P. Halmos, Measure theory, Van Nostrand, New York, 1950.

4. E. Hopf, Über die Anfangswertaufgabe für die hydrodynamischen Grundgleichungen, Math. Nachr. 4 (1951), 213-231.

5. M. Riesz, Sur les ensembles compacts de fonctions sommables, Acta. Sci. Math Szeged 6 (1933), 136-142.

UNIVERSITY OF MinNESOTA

\section{ON THE BASIS PROBLEM IN NORMED SPACES ${ }^{1}$}

\section{MAHLON M. DAY}

Since Schauder's fundamental question of the paper [9]-Does every separable Banach space have a basis?-is still unanswered, we give in this note a proof that every infinite-dimensional Banach space has a closed linear subspace which has a basis with certain extra properties. Banach [1] asserts without proof the existence of a subspace with a basis. Gelbaum [4] proves the existence of a subspace which has a basis with slightly weaker extra properties than those proved in this note. The present theorem was stated, with a few words about the proof, in my book [3, p. 72], but repeated requests for copies of a paper in which proof is given suggest that that outline is too obscure.

Indeed, when this note was first sent off, the referee discovered that the proof, given there as sketched in [3], was incorrect. We give here a proof based on a generalization of the Borsuk-Ulam theorem. The other "proof" misquoted a theorem of E. Michael in an attempt to reduce the generalization to the original theorem.

THEOREM. Let $N$ be an infinite-dimensional normed space. Then there exist biorthogonal sequences $\left(b_{i}, i \in \omega\right)$ and $\left(\beta_{i}, i \in \omega\right)$ in $B$ and $B^{*}$ respectively, such that

(i) $\left(b_{i}\right)$ is a basis for the closed linear manifold $L$ in $B$ spanned by the set of all $b_{i}$.

Received by the editors April 27, 1961 and, in revised form, May 19, 1961.

1 This note was written while the author held a Senior Postdoctoral Fellowship of the National Science Foundation (U.S.A.). 